\title{
Hyaluronan deposition and correlation with inflammation in a murine ovalbumin model of asthma
}

\author{
Georgiana Cheng ${ }^{1}$, Shadi Swaidani ${ }^{1}$, Manisha Sharma ${ }^{1}$, Mark E. Lauer ${ }^{2}$, Vincent C. \\ Hascall $^{2}$, and Mark A. Aronica ${ }^{1,3,{ }^{*}}$ \\ ${ }^{1}$ Department of Pathobiology, Cleveland Clinic, Cleveland, Ohio \\ ${ }^{2}$ Department of Biomedical Engineering, Cleveland Clinic, Cleveland, Ohio \\ ${ }^{3}$ Lerner Research Institute and the Respiratory Institute, Cleveland Clinic, Cleveland, Ohio
}

\begin{abstract}
Asthma is a chronic inflammatory disease of the airways characterized by airway remodeling, which includes changes in the extracellular matrix (ECM). However the role of the ECM in mediating these changes is poorly understood. Hyaluronan (HA), a major component of the ECM, has been implicated in asthma as well as in many other biological processes. Our study investigates the processes involved in HA synthesis, deposition, localization and degradation during an acute and chronic murine model of ovalbumin (OVA)-induced allergic pulmonary inflammation. Mice were sensitized, challenged to OVA and sacrificed at various time points during an 8-week challenge protocol. Bronchoalveolar lavage (BAL) fluids, blood, and lung tissue were collected for study. RNA, HA, protein and histopathology were analyzed. Analyses of lung sections and BAL fluids revealed an early deposition and an increase in HA levels within 24 hours of antigen exposure. HA levels peaked at day 8 in BAL, while inflammatory cell recovery peaked at day 6. Hyaluronan synthase (HAS) 1 and HAS2 on RNA levels peaked within 2 hours of antigen exposure, while hyaluronidase (HYAL)1 and HYAL2 on RNA levels decreased. Both inflammatory cell infiltrates and collagen deposition co-localized with HA deposition within the lungs. These data support a role for HA in the pathogenesis of inflammation and airway remodeling in a murine model of asthma. HA deposition appears largely due to up regulation of HAS1 and HAS2. In addition, HA appears to provide the scaffolding for inflammatory cell accumulation as well as for new collagen synthesis and deposition.
\end{abstract}

\section{Keywords}

hyaluronan; inflammation; asthma; extracellular matrix

\section{Introduction}

Asthma is a complex response in which the genetic background and the immune system interact with environmental factors to elicit the overt manifestations of the disease.

(C) 2011 Elsevier B.V. All rights reserved.

*Corresponding author: Mark Aronica, M.D., Department of Pathobiology, Cleveland Clinic, 9500 Euclid Ave, Cleveland, OH 44195 , Phone: 216-445-3080, Fax: 216-636-0104, aronicm@ccf.org.

Publisher's Disclaimer: This is a PDF file of an unedited manuscript that has been accepted for publication. As a service to our customers we are providing this early version of the manuscript. The manuscript will undergo copyediting, typesetting, and review of the resulting proof before it is published in its final citable form. Please note that during the production process errors may be discovered which could affect the content, and all legal disclaimers that apply to the journal pertain. 
Recruitment of inflammatory cells into the lungs and airway remodeling are key components in the pathogenesis of asthma. Inflammatory cells that infiltrate or are present in the lungs during an asthmatic episode include eosinophils, T lymphocytes, monocytes/ macrophages and mast cells. As a consequence of the chronic inflammation, lung morphology eventually becomes altered even in mild forms of the disease. Among the changes that are frequently noted are mucous metaplasia, smooth muscle hypertrophy, subepithelial fibrosis, increased formation of post capillary venules that are indicative of angiogenesis, and extracellular matrix (ECM) deposition (Chiappara et al., 2001; Elias et al., 2003).

In the lung, the ECM has traditionally been considered to be inert scaffolding, having only a mechanical role in supporting and maintaining tissue structure. However, recent findings indicate that the role of ECM molecules is much broader than previously thought. The ECM is in a dynamic state, and the estimated physiologic turnover of total ECM in the human lung is 10-15\%/day (Johnson, 2001). Molecules in the ECM have roles in cell-cell interactions, cell-matrix interactions, cell proliferation, cell locomotion and inflammation (Raines, 2000; Streuli, 1999). The ECM can also modulate fluid balance, elasticity, and act as a reservoir for cytokines, chemokines and growth factors (Vaday and Lider, 2000). Increased hyaluronan (HA) and proteoglycan depositions in the submucosa are also a characteristic of the asthmatic airways (Huang et al., 1999; Roberts, 1995), and it is thought that the accumulation of these molecules compromises the biomechanical properties of the airway tissue (Bosse et al., 2008; Roberts, 1995). However, little is known about how ECM molecules affect the pathology of asthma (Yamauchi and Inoue, 2007).

HA is a large glycosaminoglycan in which the disaccharide (glucuronic acid- $\beta 1,3-\mathrm{N}$ acetylglucosamine- $\beta 1,4-$ ) is repeated several thousand times (Fraser et al., 1997). HA is produced by almost all vertebrate cells and is a ubiquitous component of connective tissues (Fraser et al., 1997). HA is synthesized by one or more of the three eukaryotic hyaluronan synthases (HAS1, 2, and 3) at the cytosolic side of the plasma membrane and is simultaneously extruded into the extracellular space (Weigel and DeAngelis, 2007). Conversely, HA is primarily degraded by hyaluronidase (HYAL)1 and HYAL2 (Stern and Jedrzejas, 2006). In addition to the normal turnover and regulation of HA, other factors such as activation of matrix metalloproteinases (MMPs) (Isnard et al., 2001) and reactive oxygen species (Tammi et al., 2002) can significantly alter the balance and turnover of HA. Outside the cell, HA interacts with both cell surface and extracellular hyaluronan-binding proteins (Day and Prestwich, 2002), providing the tissue with a structural scaffold. In addition to its function in matrix organization, HA has roles in many physiologic and pathologic processes that depend on cell migration, including morphogenesis, tissue regeneration, wound repair, as well as tumor cell growth and invasion (Fraser et al., 1997).

There is now mounting evidence that HA has an active role in the pathobiology of asthma. HA was first noted in the secretions of asthmatics in 1978 (Sahu and Lynn, 1978), and HA levels in the bronchoalveolar lavage fluid (BAL) fluid of asthmatics have been associated with the severity of disease (Bousquet et al., 1991). Increased amounts of HA are found in the asthmatic airway submucosa and around smooth muscle bundles, where it is hypothesized to have a role in the increased airway smooth muscle cell growth seen in asthmatics (Roberts, 1995). While HA in the BAL may have a beneficial role by retaining enzymes important for host defense (Forteza et al., 2001), accumulation of excess HA in the submucosal tissue can lead to severe airway obstruction and death as shown in a model of bleomycin-induced lung injury in CD44 null mice (Teder et al., 2002). HA is also an important co-stimulatory factor for the differentiation and proliferation of eosinophils in vitro (Hamann et al., 1995) and has also been implicated in increasing eosinophil survival as well as enhancing TGF- $\beta$, a cytokine linked to airway remodeling (Ohkawara et al., 2000). 
Thus, while the trafficking of inflammatory cells is clearly dependent on integrins, selectins and cytokine/chemokine gradients, the hyaluronan-based ECM defines where the problem is locally and subsequently promotes adhesion and likely subsequent activation of the inflammatory cells.

Although HA has been linked to asthma, little is known about HA in asthma with regards to production, deposition, interaction with inflammatory cells, or interaction with other matrix components. While no animal model recapitulates all aspects of the human disease, animal models have formed the basis for much of our current understanding of the pathophysiology of asthma (Bates et al., 2009; Boyce and Austen, 2005). Similarities include the development of allergen-induced airway hyperresponsiveness, smooth muscle hypertrophy, subepithelial fibrosis, as well as goblet cell metaplasia, reviewed in (Boyce and Austen, 2005). Based on these observations, we utilized a time course analysis to delineate HA synthesis, production, and degradation in the lung using an acute and chronic murine model of antigen-induced pulmonary inflammation.

\section{Results}

\subsection{Hyaluronan Deposition and Content in the Lung Increases with Allergen Challenge}

Given the increasingly recognized roles of HA in disease processes and the evidence suggesting that HA has an active role in asthma pathogenesis, we first investigated the deposition and content of HA in a model of antigen induced pulmonary inflammation. After initial sensitization, see methods section for details, mice were exposed to repeated OVA inhalations, once daily for 8 days (acute phase) followed by twice weekly exposure for 7 additional weeks (chronic phase). Confocal imaging demonstrated that naïve mice have structural HA supporting the airways and blood vessels with some noticeable HA in the lung parenchyma as well (Fig. 1, naive). During antigen challenge, increased HA was observed as early as $12 \mathrm{hrs}$ after the initial challenge with peak levels seen at days 6 and 8 . The increased HA is maintained for 2 weeks, diminishes at 4 and 6 weeks with what appears to be near baseline levels at 8 weeks (Fig. 1). HA levels in the lung tissue were analyzed by Fluorophore-assisted carbohydrate electrophoresis (FACE) analysis as described in Methods. HA content was also increased as early as $12 \mathrm{hrs,} \mathrm{increased} \mathrm{further} \mathrm{at} \mathrm{day} \mathrm{one,}$ peaked at day 6, remained elevated thru week 2 and decreased during weeks 4 thru 6 (Fig. 2A). There are statistically significant increase from 12 hrs through 6 weeks compared with naïve mice. The HA content is similar to what was seen in the confocal images (Fig. 1). HA was first noted in the secretions of asthmatics in 1978 (Sahu and Lynn, 1978), and HA levels in the BAL fluid of asthmatics has been associated with the severity of the disease (Bousquet et al., 1991). Therefore, we determined the HA content of the BAL fluid in mice undergoing allergen challenge. There was an early but not statistically significant rise in HA levels within 6 and $12 \mathrm{hrs}$ of the first antigen challenge with statistical significance seen by day 1 (Fig. 2B). HA continues to increase to a peak at day 8 , decreases substantially by week 2 , and returns to near baseline levels by week 8 (Fig. 2B).

\subsection{Increased Expression of Hyaluronan Synthases During Antigen Challenge}

HA is synthesized by one or more of the three eukaryotic hyaluronan synthases (HAS1, 2 and 3) (Weigel and DeAngelis, 2007) and can be degraded by the hyaluronidases (HYAL1 and HYAL2) (Stern and Jedrzejas, 2006). Given the increase in HA content in both the lung and BAL fluid, we determined mRNA changes of these enzymes by qPCR in the lung of antigen challenged mice. We found that all three of the HAS enzymes are expressed in the naïve lung, but the level of expression varied significantly with HAS1 > HAS2 > HAS 3 (18:1.3:1, data not shown). During antigen challenge both HAS1 and HAS2 mRNA increased rapidly, within $2 \mathrm{hrs}$ (Fig. 3A). Interestingly, while HAS1 returned to baseline 
levels within day 1 of first antigen exposure (Fig. 3A, circles, left axis), HAS2 levels remained elevated during the acute challenge and gradually decreased to baseline levels by day 8 (Fig. 3A, squares, right y-axis). HAS3 expression did not change during the acute or chronic antigen exposure phase (data not shown). Of note, HAS1 RNA levels at 2, 4 and 6 hrs after OVA aerosol challenge on day 6 did not increase, suggesting that the early increase was associated only with the very first antigen exposure and not with subsequent exposures (data not shown). In the OVA challenge only group i.e. without sensitization, HAS1 and HAS2 did not show increase at 2 and 6 hrs (data not shown). In contrast to the increase in RNA levels seen for HAS1 and HAS2, HYAL1 and HYAL2 mRNA expression levels decreased within $12 \mathrm{hrs}$ of the first antigen exposure and remained below baseline levels until late in the chronic phase (Fig. 3B). Taken together, these data suggest that the induction of HAS1 might be responsible for very early phase HA production while HAS2 might be responsible for early and late phase HA production, and with the concomitant reduction in HYAL1 and HYAL2, are responsible for HA accumulation in the lung in this model.

\subsection{HA Deposition in the Lung Co-localizes with Inflammation and New Collagen Synthesis}

BAL fluid was analyzed in order to determine if the HA levels correlated with the influx of inflammatory cells. BAL cellularity increased as early as $6 \mathrm{hrs}$ after the first OVA aerosol exposure and continued to increase to a peak at day 6 (Fig. 4A). BAL cellularity then decreased, but remained higher than baseline during the chronic phase, weeks $2-8$. Eosinophils in BAL showed a similar pattern during the acute challenge, given that the majority of total nucleated cells in the BAL are eosinophils (Fig. 4B), but decreased substantially during the chronic phase. Lymphocytes in BAL showed a similar pattern as eosinophils (Fig. 4C) while macrophages/monocytes did not show same trend as Eosinophils and lymphocytes (Fig. 4D). H\&E staining of lung sections revealed that the inflammatory cells accumulate primarily in the perivascular and peribronchial areas as shown by the clusters of blue stained mononuclear cells (Fig. 5, lower magnifications in Supplement fig. 1), which is typical in our murine model of antigen-induced pulmonary inflammation (Aronica et al., 1999).

To better characterize the influx and location of eosinophils, we stained lung sections with eosinophil major basic protein (MBP). Analysis of lung sections reveals the presence of eosinophils scattered in the lung as early as 12 hrs (Fig. 6 showing MBP stain (red), Supplement Fig. 2). The eosinophils then localized in the perivascular and peribronchial areas from day 2 through week 2 , and then they dissipated and were no longer present by week 6 (Fig. 6, Supplement Fig. 2). Staining for both HA (green) and MBP (red) showed that eosinophils that entered the lung were distributed throughout the lung by $12 \mathrm{hrs}$ (Fig. 6) even though early increases in HA are already apparent in the peribronchial area. However, by day 2 many of the eosinophils were now co-localized with HA in the perivascular and peribronchial areas (Fig. 6). This co-localization pattern continued throughout the remainder of the time course (Fig. 6A and B) until the eosinophils begin to dissipate after week 2 (Fig. 6). Quantifying HA and eosinophils by Image-Pro plus 7.0 software showed the same trend (Supplement Fig. 3B and 3C) as the staining pattern, with significant correlation between HA and eosinophils (Supplement Fig. 3A).

Increased collagen synthesis is a key feature of human asthma and is also noted in murine models of antigen-induced pulmonary inflammation. Therefore, we stained lung sections for both HA (green) and collagen (red). HA and collagen deposition co-localize as early as 12 hrs after the first OVA aerosol exposure (Fig. 7, lower magnifications in Supplement Fig. 4). Collagen also accumulates in the perivascular and peribronchial areas that in higher power views appears to be intertwined with HA (Fig. 7A). Quantifying HA and collagen by Image- 
Pro plus 7.0 software showed the same trend (Fig. 7B), and a significant correlation between HA and collagen (Fig. 7B). Taken together these data show that HA deposition in the lung in response to the OVA challenge is closely linked to inflammatory cell localization as well as collagen deposition.

\section{Discussion}

In this study we utilized an acute and chronic model of antigen-induced pulmonary inflammation to better understand the factors that control the synthesis, deposition and turnover of HA in the asthmatic airway. In addition we utilized confocal microscopy to gain a clearer understanding of HA deposition in relation to inflammatory cell recruitment and collagen deposition in the lung.

We have shown that HA deposition in the lung occurs as early as $12 \mathrm{hrs}$ after the first OVA aerosol challenge (Fig.1), and significantly increased levels of HA in the lung and BAL fluid can be detected by 1 day after the initial OVA aerosol challenge and remain elevated for at least 2 weeks during chronic antigen challenge (Figs. 2A and B). Increased HA was seen in the perivascular and peribronchial areas, which are the same areas in which inflammatory cells localize (Figs. 1 and Supplement Fig. 2A). The increased mRNA levels of both HAS1 and HAS2 within 2 hrs of antigen exposure suggest that these synthases are responsible for the rapid increase in HA. Conversely, the decline in mRNA levels of the HYALs also suggests that diminished degradation may also have a role in HA accumulation within the lung parenchyma (Fig. 3B). Finally, eosinophil localization and collagen deposition within the lung parenchyma were also closely linked to HA synthesis and deposition, occurring in the same perivascular and peribronchial areas.

HA is synthesized by almost all vertebrate cells and is a ubiquitous component of connective tissue. However, existing evidence supports the notion that HA produced during pathological states is functionally distinct from the HA present in normal tissue (Milinkovic et al., 2004). Members of our group have shown that airway epithelial cells produce HA in response to endoplasmic reticulum (ER) stress and airway smooth muscle cells produce HA in response to both ER and viral poly I:C stresses (Lauer et al., 2008; Lauer et al., 2009a; Lauer et al., 2009b). The HA matrix in all of these cases is leukocyte-adhesive. In addition, the ECM, and HA in particular, can also modulate fluid balance, elasticity, and act as a reservoir for cytokines, chemokines, and growth factors (Vaday and Lider, 2000).

Based on these observations one may hypothesize the following; during the initial antigen exposure in the lungs airway epithelial cells may be the first responders and release a variety of cytokines and chemokines. Structural cells in the lung begin to produce hyaluronan in response to these signals leading to the upregulation of HAS mRNA in preparation for the sustained production of HA and begin to down regulate HYALs in order to minimize HA degradation. Eosinophils move into the lung very early but are somewhat scattered as seen in Fig. 6,12 hrs and supplemental Fig. 2, 12 hrs. The HA matrix is laid down and chemokines begin to accumulate in this gel like substance setting up a gradient for inflammatory cells to follow which leads to the co-localization of eosinophils and HA as seen in Fig. 6, later time points. The interaction of HA with eosinophils may then lead to the enhanced production and/or release of TGF-beta from these cells and begin the process of remodeling and collagen deposition (Ohkawara et al., 2000). The HA matrix may also be part of the necessary scaffolding needed for collagen deposition, leading to the colocalization of HA and collagen as seen in Fig. 7 and supplemental Fig. 4. Once the collagen is laid down, HA can then be cleared from the lung, as seen in supplemental Fig 4, at the 6 and 8 week time points. 
The mechanism by which HA synthesis is activated in this model has not yet been identified.. Prostaglandins are well known mediators in both human and murine models of asthma and may have a role in HAS induction (Fischer and Schror, 2007) as well as a variety of cytokines involved in the asthmatic response (Jiang et al., 2007; Wilkinson et al., 2004). In addition, kdel expression, a marker of ER stress, can be seen in murine models of asthma (A. Majors, M. Aronica and V. Hascall unpublished observations) suggesting a novel role for ER stress in the pathogenesis of asthma.

In summary, these data defined the temporal relationship between antigen exposure and the onset of HA synthesis and deposition within the lung and subsequent degradation. In addition, our data suggest that HA is a key mediator in organizing the inflammation in the characteristic perivascular and peribronchial distribution seen in this model as well as in organizing changes associated with new collagen deposition.

\section{Experimental procedures}

\subsection{Mice}

Immunocompetent, 6- to 8-wk-old female BALB/c mice from the Jackson Laboratory (Bar Harbor, ME) were used for OVA sensitization and challenge (Aronica et al., 2004). All mice were maintained in the Cleveland Clinic animal care facility in a specific pathogen-free condition using microisolator cages and were used in accordance with applicable regulations after institutional approval.

\subsection{OVA Sensitization}

Standard induction of allergic airways disease was done as previously described (Aronica et al., 2004). In brief, BALB/c mice were immunized by intraperitoneal injection (IP) with 10 $\mu \mathrm{g}$ of OVA (Sigma Chemicals), adsorbed to $20 \mathrm{mg}$ of aluminum hydroxide $\left(\mathrm{Al}(\mathrm{OH})_{3}\right)$. Two weeks later, mice were exposed to an aerosol of $1 \%$ OVA in PBS for 45 min using an Ultrasonic 2000 nebulizer (Nouvag, Switzerland). The acute phase consisted of once daily exposure for a total of 8 days followed by the chronic phase, which consisted of twice weekly exposure for a total of 7 additional weeks (total 8 week study protocol). Mice were sacrificed at the following time points after the first OVA aerosol exposure: 2, 4, 6, and 12 hrs. Mice were then sacrificed $24 \mathrm{hrs}$ after the last OVA aerosol exposure on days 1, 2, 4, 6, and 8; and on weeks 2, 4, 6, and 8. Naïve mice and IP sensitized mice ( 0 hrs) were used as controls. Four mice were used at each time point for the study.

\subsection{Collection of Blood and Bronchoalveolar Lavage Fluid}

At each time point, mice were euthanized with an IP injection of sodium pentobarbital. Blood was drawn in EDTA syringes by cardiac puncture and run on an ADVIA 120 Hematology System (Siemens Healthcare Diagnostics, IL) to obtain total cell count and differential. BAL was obtained by cannulating the trachea with a 24 guage feeding needle and lavaging the lungs with $700 \mu \mathrm{L}$ of HL-1 medium (serum-free medium). The typical BAL fluid return was $400-600 \mu \mathrm{L}$. White blood cells were counted on a hemocytometer using ethidium bromide/acridine orange staining of nucleated cells. Differential counts were based on counts of 200 cells after staining with HEMA 3 (Fisher Diagnostics, Middletown, VA) using standard morphologic criteria to classify the cells as eosinophils, lymphocytes, neutrophils or other mononuclear leukocytes (alveolar macrophages and monocytes). The remainder of each BAL sample was centrifuged for $5 \mathrm{~min}$ at $1000 \mathrm{rpm}$ at $4{ }^{\circ} \mathrm{C}$. Supernatants were transferred to a new tube and analyzed for HA with a hyaluronic acid-binding protein (HABP) ELISA-like assay. 


\subsection{Quantitation of HA by a Specific ELISA-like Assay}

Quantitation of HA was determined by using the HA test kit (Corgenix, Inc. Broomfield, $\mathrm{CO})$ according to manufacturer's instructions. The HA test kit is an enzyme-linked binding protein assay that uses a capture molecule known as HABP. Diluted BAL samples were incubated in HABP-coated microwells, allowing HA present in samples to react with the immobilized HABP. After the removal of unbound BAL molecules by washing with PBS, HABP conjugated with horseradish peroxidase (HRP) solution was added to the microwells to form complexes with bound HA. Following another washing with PBS, a chromogenic substrate of tetramethylbenzidine and hydrogen peroxide was added to develop a colored reaction. The intensity of the color was measured in optical density (O.D.) units with a spectrophotometer at $450 \mathrm{~nm}$. HA levels in BAL and control samples were determined against a reference curve prepared from the reagent blank and the HA reference solution provided with the kit.

\subsection{Fluorophore-assisted Carbohydrate Electrophoresis}

Preparation of HA for Fluorescent Derivatization with 2-Aminoacridone - The fluorophoreassisted carbohydrate electrophoresis (FACE) method for the quantification of HA has been described (Lauer et al., 2009b). Briefly, the lower lobe of right lung was digested with proteinase $\mathrm{K}$ (Invitrogen, Carlsbad, CA) at $1 \mathrm{mg} / \mathrm{ml}$ in $100 \mathrm{mM}$ ammonium acetate, $\mathrm{pH} 7.0$ with $0.01 \%$ lauryl sulfate at $60{ }^{\circ} \mathrm{C}$ for $4 \mathrm{hrs}$, and $\mathrm{HA}$ was recovered by ethanol precipitation. The HA was digested to disaccharides at $37^{\circ} \mathrm{C}$ overnight with hyaluronidase SD at $2.5 \mathrm{mU} /$ $\mu \mathrm{l}$ (Seikagaku) and chondroitinase $\mathrm{ABC}$ at $25 \mathrm{mU} / \mu \mathrm{l}$ (Seikagaku) and labeled with 2aminoacridone (AMAC) (Invitrogen). The samples were electrophoresed on a polyacrylamide gel, and the gel-plates were washed with distilled water. After electrophoresis, the gels (in their glass plates) were placed on a UV transilluminator (Ultra Lum, Claremont, CA) and illuminated at $365 \mathrm{~nm}$. Imaging was captured on a Quantix CCD camera (Photometrics, Tucson, AZ), and the HA disaccharide band was quantified using Gel-Pro Analyzer® version 3.0 (Media Cybernetics, Silver Spring, MD). All statistics (student t-tests) were done using KaleidaGraph v3.6 (Synergy Software, Reading, PA).

The lower lobes of the right lungs from six mice were also collected and lyophilized to obtain the dry weight of the lung tissue. The total amount $(\mu \mathrm{g})$ of HA from each lower lobe of right lung was calculated per dry weight (d.w.).

\subsection{Lung Collection and Histology}

Superior and middle lobes of the right lung were collected for RNA preparation, and lower lobes of right and left lungs were collected for HA and protein preparation. The left upper lobe of the lung was collected for histology by fixation in $10 \%$ formalin overnight and then paraffin-embedded or for immunohistochemistry by inflating the left upper lobe with optimal cutting temperature (OCT) compound (Sakura Finetek, Torrance, CA) and then OCT-embedded and stored at $-80^{\circ} \mathrm{C}$.

Paraffin embedded lung tissue was sectioned at $5 \mu \mathrm{m}$ and stained with hematoxylin and eosin (H\&E) to determine morphology and inflammatory cell infiltrate. OCT embedded fresh frozen lung tissue was sectioned at $8 \mu \mathrm{m}$ using a cryostat for immunohistochemistry analyses.

\subsection{Immunohistochemistry}

Fresh frozen sections were fixed in cold acetone/methanol 50/50\% for $10 \mathrm{~min}$. Sections were then blocked with 3\% BSA/PBS for all antibodies used. In addition, following 3\% BSA/PBS blocking, sections were blocked with avidin, biotin, and streptavidin (Vector Laboratories, Burlingame, CA) for all HABP staining or for double staining with HABP. 
Sections were incubated with biotinylated HABP (Seikagaku, $5 \mu \mathrm{g} / \mathrm{ml}$ ) or the primary antibody for $1 \mathrm{hrs}$ at room temperature or overnight at $4{ }^{\circ} \mathrm{C}$. Primary antibodies were goat polyclonal antibody against eosinophil major basic protein (EMBP) (Santa Cruz 1:50 dilution), and polyclonal goat anti-human collagen III (Southern Biotechnology Associates, Inc., Birmingham, AL, 1:10 dilution). After washing, secondary antibody was added and incubated for $1 \mathrm{hrs}$ at room temperature in the dark. Streptavidin conjugated 488 (for HABP) or donkey anti goat 594 (for EMBP or collagen III) were used as secondary antibodies. Each staining included a control by using only the secondary antibody to demonstrate the specificity of antibody staining. All of these secondary antibody only controls were negative. Sections were examined with a Leica fluorescence microscope, and images were captured with a Retiga 2000R camera and QCapture pro6 software.

HABP for HA staining, EMBP for eosinophils staining, and collagen III for collagen staining in lung tissues were quantitated by Image-Pro plus 7.0 software.

\subsection{Real-Time Quantitative PCR (qRT-PCR) Technique}

Total RNA was extracted from lung tissue using a standardized TRIzol method of phenol extraction (Invitrogen, Carlsbad, CA) and then cleaned with RNA cleanup kit (Qiagen, Valencia, CA). Total RNA was measured by NanoDrop (Wilmington, DE). The A260/A280 ratios were between 1.9 and 2.2 for all samples. Total RNA samples were pooled together for each time point for qRT-PCR analysis. Transcripts were studied by qRT-PCR in triplicate. Transcript-specific primers were generated based on mouse sequences from GenBank and were designed using Primer Express software (Applied Biosystems, Inc. ABI, Foster City, CA). NCBI BLAST was used to ensure specificity of each primer pair for each transcript. All primers were checked for dissociation curves and melting temperatures. Primer sequences are listed in Table 1. Briefly, reverse transcription was done using Superscript ${ }^{\mathrm{TM}}$ First-Strand Synthesis System for RT-PCR (Invitrogen, Carlsbad, CA) to generate first strand cDNA according to the manufacturer's instruction. qRT-PCR was done with SYBR green PCR Master mix (Applied Biosystems, Foster City, CA) using an ABI 7300 sequence detection instrument (Applied Biosystems, Foster City, CA). Mouse glyceraldehyde phosphate dehydrogenase (GAPDH) was used as an internal control, and relative transcript abundance was normalized to the amount of GAPDH for the qRT-PCR data. Mean fold changes were calculated by averaging the triplicate measurements for each gene. The relative fold difference calculation uses the $2-\Delta \Delta \mathrm{CT}$ method.

\subsection{Statistical Analysis}

Data were analyzed and tested by Graph Pad Prism software 5 (GraphPad Software, La Jolla, CA) for statistical significance $(\mathrm{p}<0.05)$ using nonparametric tests unpaired $\mathrm{t}$ tests with two-tails. Results are presented as the mean \pm standard error of the mean (SEM).

\section{Supplementary Material}

Refer to Web version on PubMed Central for supplementary material.

\section{Acknowledgments}

Supported by Grants: This work was supported by National Institute of Health Grants AI067816 (M.A.A) and HL081064 (V.C.H. and M.A.A)

\section{Abbreviations used}

BAL Bronchoalveolar lavage 


$\begin{array}{ll}\text { ECM } & \text { Extracellular matrix } \\ \text { HA } & \text { Hyaluronan } \\ \text { HAS } & \text { Hyaluronan synthase } \\ \text { HYAL } & \text { Hyaluronidase } \\ \text { IP } & \text { Intraperitoneal } \\ \text { MBP } & \text { Major basic protein } \\ \text { OVA } & \text { Ovalbumin }\end{array}$

\section{References}

Aronica MA, McCarthy S, Swaidani S, Mitchell D, Goral M, Sheller JR, Boothby M. Recall helper T cell response: T helper 1 cell-resistant allergic susceptibility without biasing uncommitted CD4 T cells. Am J Respir Crit Care Med. 2004; 169:587-595. [PubMed: 14617509]

Aronica MA, Mora AL, Mitchell DB, Finn PW, Johnson JE, Sheller JR, Boothby MR. Preferential role for NF-kappa B/Rel signaling in the type 1 but not type $2 \mathrm{~T}$ cell-dependent immune response in vivo. J Immunol. 1999; 163:5116-5124. [PubMed: 10528218]

Bates JH, Rincon M, Irvin CG. Animal models of asthma. Am J Physiol Lung Cell Mol Physiol. 2009; 297:L401-L410. [PubMed: 19561139]

Bosse Y, Pare PD, Seow CY. Airway wall remodeling in asthma: from the epithelial layer to the adventitia. Curr Allergy Asthma Rep. 2008; 8:357-366. [PubMed: 18606090]

Bousquet J, Chanez P, Lacoste JY, Enander I, Venge P, Peterson C, Ahlstedt S, Michel FB, Godard P. Indirect evidence of bronchial inflammation assessed by titration of inflammatory mediators in BAL fluid of patients with asthma. J Allergy Clin Immunol. 1991; 88:649-660. [PubMed: 1918730]

Boyce JA, Austen KF. No audible wheezing: nuggets and conundrums from mouse asthma models. J Exp Med. 2005; 201:1869-1873. [PubMed: 15967817]

Chiappara G, Gagliardo R, Siena A, Bonsignore MR, Bousquet J, Bonsignore G, Vignola AM. Airway remodelling in the pathogenesis of asthma. Curr Opin Allergy Clin Immunol. 2001; 1:85-93. [PubMed: 11964675]

Day AJ, Prestwich GD. Hyaluronan-binding proteins: tying up the giant. J Biol Chem. 2002; 277:4585-4588. [PubMed: 11717315]

Elias JA, Lee CG, Zheng T, Ma B, Homer RJ, Zhu Z. New insights into the pathogenesis of asthma. J Clin Invest. 2003; 111:291-297. [PubMed: 12569150]

Fischer JW, Schror K. Regulation of hyaluronan synthesis by vasodilatory prostaglandins. Implications for atherosclerosis. Thromb Haemost. 2007; 98:287-295. [PubMed: 17721609]

Forteza R, Lieb T, Aoki T, Savani RC, Conner GE, Salathe M. Hyaluronan serves a novel role in airway mucosal host defense. FASEB J. 2001; 15:2179-2186. [PubMed: 11641244]

Fraser JR, Laurent TC, Laurent UB. Hyaluronan: its nature, distribution, functions and turnover. J Intern Med. 1997; 242:27-33. [PubMed: 9260563]

Hamann KJ, Dowling TL, Neeley SP, Grant JA, Leff AR. Hyaluronic acid enhances cell proliferation during eosinopoiesis through the CD44 surface antigen. J Immunol. 1995; 154:4073-4080. [PubMed: 7535820]

Huang J, Olivenstein R, Taha R, Hamid Q, Ludwig M. Enhanced proteoglycan deposition in the airway wall of atopic asthmatics. Am J Respir Crit Care Med. 1999; 160:725-729. [PubMed: 10430752]

Isnard N, Legeais JM, Renard G, Robert L. Effect of hyaluronan on MMP expression and activation. Cell Biol Int. 2001; 25:735-739. [PubMed: 11482897]

Jiang D, Liang J, Noble PW. Hyaluronan in tissue injury and repair. Annu Rev Cell Dev Biol. 2007; 23:435-461. [PubMed: 17506690] 
Johnson PR. Role of human airway smooth muscle in altered extracellular matrix production in asthma. Clin Exp Pharmacol Physiol. 2001; 28:233-236. [PubMed: 11236132]

Lauer ME, Erzurum SC, Mukhopadhyay D, Vasanji A, Drazba J, Wang A, Fulop C, Hascall VC. Differentiated murine airway epithelial cells synthesize a leukocyte-adhesive hyaluronan matrix in response to endoplasmic reticulum stress. J Biol Chem. 2008; 283:26283-26296. [PubMed: 18644783]

Lauer ME, Fulop C, Mukhopadhyay D, Comhair S, Erzurum SC, Hascall VC. Airway smooth muscle cells synthesize hyaluronan cable structures independent of inter-alpha-inhibitor heavy chain attachment. J Biol Chem. 2009a; 284:5313-5323. [PubMed: 19075022]

Lauer ME, Mukhopadhyay D, Fulop C, de la Motte CA, Majors AK, Hascall VC. Primary murine airway smooth muscle cells exposed to poly(I,C) or tunicamycin synthesize a leukocyte-adhesive hyaluronan matrix. J Biol Chem. 2009b; 284:5299-5312. [PubMed: 19088077]

Milinkovic M, Antin JH, Hergrueter CA, Underhill CB, Sackstein R. CD44-hyaluronic acid interactions mediate shear-resistant binding of lymphocytes to dermal endothelium in acute cutaneous GVHD. Blood. 2004; 103:740-742. [PubMed: 14504094]

Ohkawara Y, Tamura G, Iwasaki T, Tanaka A, Kikuchi T, Shirato K. Activation and transforming growth factor-beta production in eosinophils by hyaluronan. Am J Respir Cell Mol Biol. 2000; 23:444-451. [PubMed: 11017908]

Raines EW. The extracellular matrix can regulate vascular cell migration, proliferation, and survival: relationships to vascular disease. Int J Exp Pathol. 2000; 81:173-182. [PubMed: 10971738]

Roberts CR. Is asthma a fibrotic disease? Chest. 1995; 107:111S-117S. [PubMed: 7874987]

Sahu S, Lynn WS. Hyaluronic acid in the pulmonary secretions of patients with asthma. Biochem J. 1978; 173:565-568. [PubMed: 697736]

Stern R, Jedrzejas MJ. Hyaluronidases: their genomics, structures, and mechanisms of action. Chem Rev. 2006; 106:818-839. [PubMed: 16522010]

Streuli C. Extracellular matrix remodelling and cellular differentiation. Curr Opin Cell Biol. 1999; 11:634-640. [PubMed: 10508658]

Tammi MI, Day AJ, Turley EA. Hyaluronan and homeostasis: a balancing act. J Biol Chem. 2002; 277:4581-4584. [PubMed: 11717316]

Teder P, Vandivier RW, Jiang D, Liang J, Cohn L, Pure E, Henson PM, Noble PW. Resolution of lung inflammation by CD44. Science. 2002; 296:155-158. [PubMed: 11935029]

Vaday GG, Lider O. Extracellular matrix moieties, cytokines, and enzymes: dynamic effects on immune cell behavior and inflammation. J Leukoc Biol. 2000; 67:149-159. [PubMed: 10670574]

Weigel PH, DeAngelis PL. Hyaluronan synthases: a decade-plus of novel glycosyltransferases. J Biol Chem. 2007; 282:36777-36781. [PubMed: 17981795]

Wilkinson TS, Potter-Perigo S, Tsoi C, Altman LC, Wight TN. Pro- and anti-inflammatory factors cooperate to control hyaluronan synthesis in lung fibroblasts. Am J Respir Cell Mol Biol. 2004; 31:92-99. [PubMed: 14764429]

Yamauchi K, Inoue H. Airway remodeling in asthma and irreversible airflow limitation-ECM deposition in airway and possible therapy for remodeling. Allergol Int. 2007; 56:321-329. [PubMed: 17965575] 

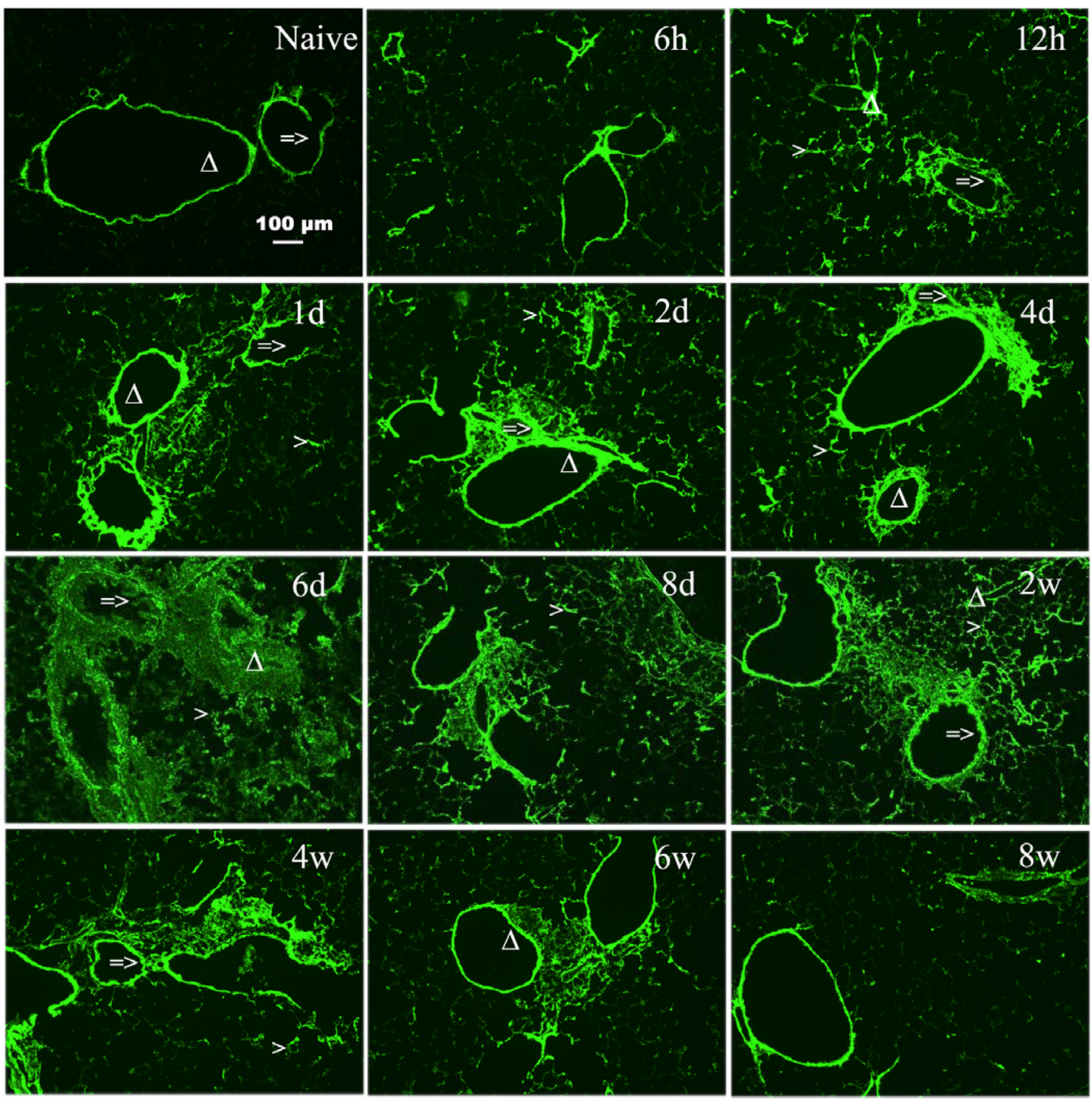

Figure 1. Increased HA accumulation in OCT sections from lungs isolated during acute and chronic stages of antigen exposure

Sections were stained with the HABP as described in Methods (magnification bar $100 \mu \mathrm{m}$ ). HA deposition is shown for lungs from naïve (unchallenged) mouse and from OVAsensitized mice during the course of allergen challenge. Mice were sacrificed at the following time points during the course of antigen exposure at 6 hours (6h), 12 hours (12h), 1 day (1d), 2 days (2d), 4 days (4d), 6 days (6d), 8 days (8d), 2 weeks (2w), 4 weeks (4w), 6 weeks $(6 \mathrm{w})$, and 8 weeks $(8 \mathrm{w})$.

$\Rightarrow$ indicates the airway of lung section.

$\Delta$ indicates the blood vessel of lung section. 
> indicates the alveolar interstitium of lung section 


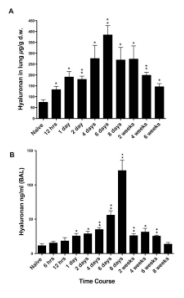

Figure 2. Quantitation of $\mathrm{HA}$ in lung tissue and in BAL during acute and chronic stages of antigen exposure

(A) HA in lung tissue was measured by the FACE method and normalized to dry weight (d.w.). (B) HA in BAL was measured by the HABP ELISA-like method. Mice were sacrificed at the indicated time points during the course of antigen exposure as in figure 1. Data represent mean values \pm SEM for the amount of HA in the lung in micrograms per gram of lung dry weight $(\mu \mathrm{g} / \mathrm{g}$ d.w. $)(n=3)$ and HA in the BAL $(n g / m l)(n=4)$.

$* \mathrm{p}<0.05$ compared with naïve mice

$* * \mathrm{p}<0.01$ compared with naïve mice 

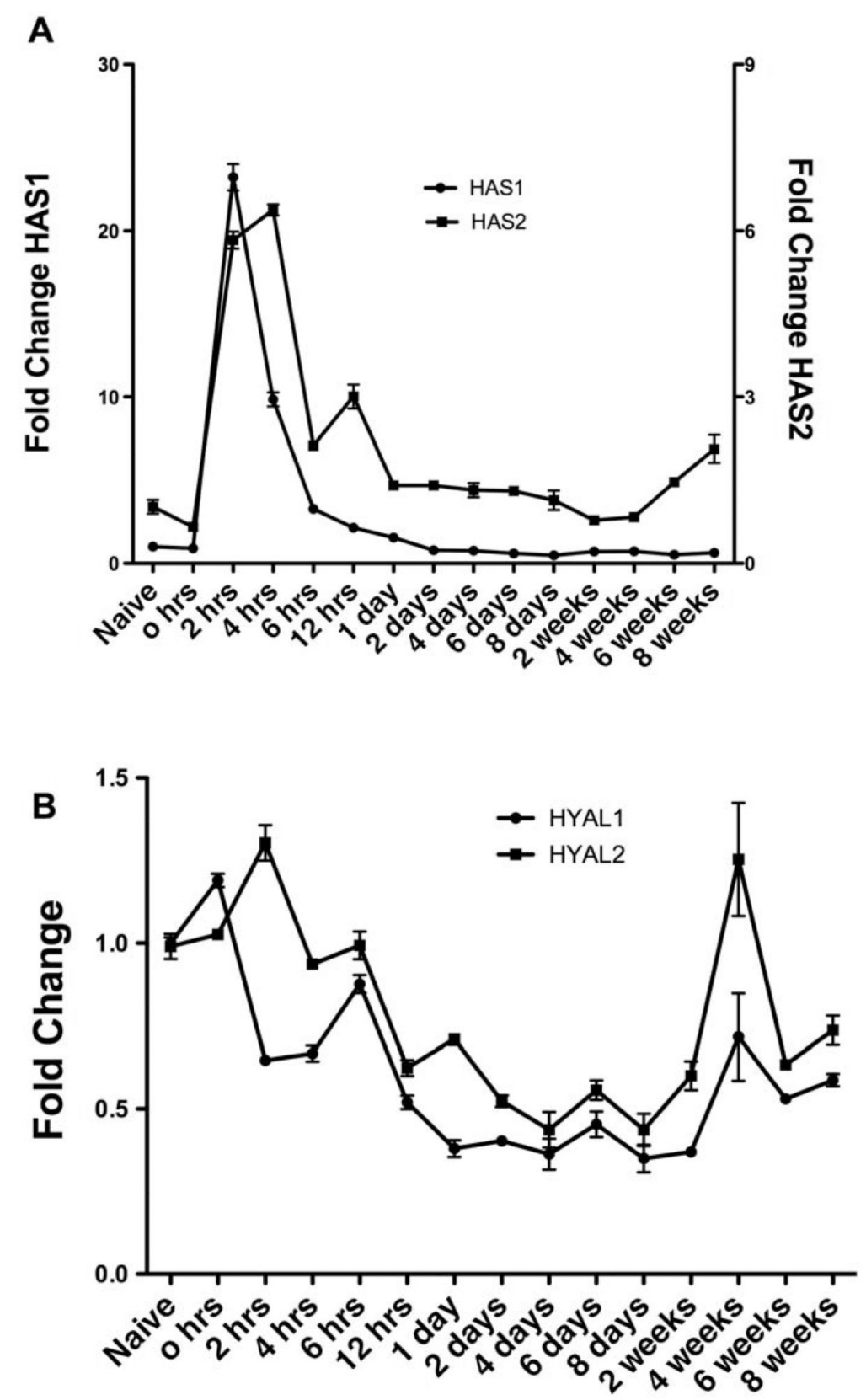

Time Course

Figure 3. qPCR analyses of mRNA expression levels for HA synthases (HAS) and hyaluronidases (HYAL) in lung tissue isolated at different times of antigen exposure

(A) The changes in HAS1 and HAS2 mRNA levels relative to values from the naïve lung are shown. Data represent mean values of fold changes on the right $y$-axis for HAS2. (B)

The changes in HYAL1 and HYAL2 mRNA levels relative to values from the naïve lung are shown. Mice were sacrificed at the indicated time points during the course of antigen exposure as in figure 1. 


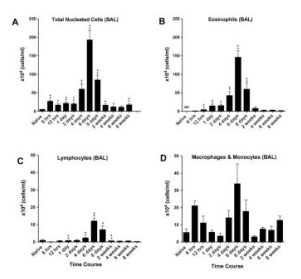

Figure 4. Total nucleated cells and eosinophils, lymphocytes, macrophages in BAL during the course of antigen exposure

(A) Total nucleated cells recovered. (B) Eosinophils recovered. (C) Lymphocytes recovered. (D) Macrophages/Monocytes recovered. Mice were sacrificed at the indicated time points during the course of antigen exposure as in figure 1. Data represent mean values \pm SEM $(\mathrm{n}=4)$.

$* \mathrm{p}<0.05$ compared with naïve mice

$* * \mathrm{p}<0.01$ compared with naïve mice 


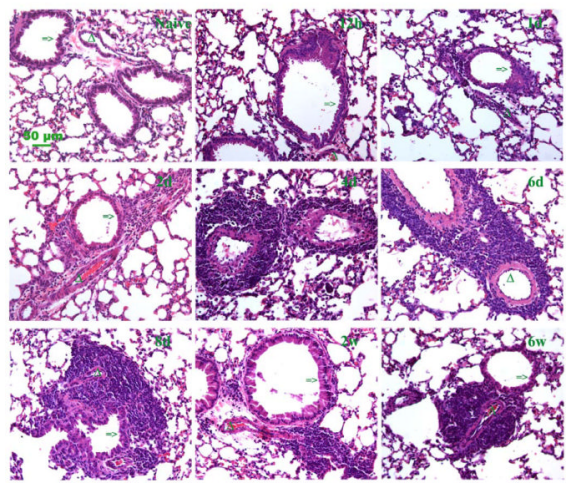

Figure 5. Histopathologic changes in lung sections during acute and chronic stages Hematoxylin and eosin (H\&E) stained paraffin sections represent the inflammation infiltrate trend for the acute stage (12 h, 1 and 2 days) and chronic stage (4 days through 6 weeks) of antigen exposure. Clumping cells demonstrate the progression of perivascular and peribronchial pulmonary inflammation (magnification bar $50 \mu \mathrm{m}$ ). A section from a control lung (naïve) is also shown. Additional low magnification fields are shown in supplement figure 1 . Mice were sacrificed at the time points during the course of antigen exposure as in figure 1.

$\Rightarrow$ indicates the airway of lung section.

$\Delta$ indicates the blood vessel of lung section. 


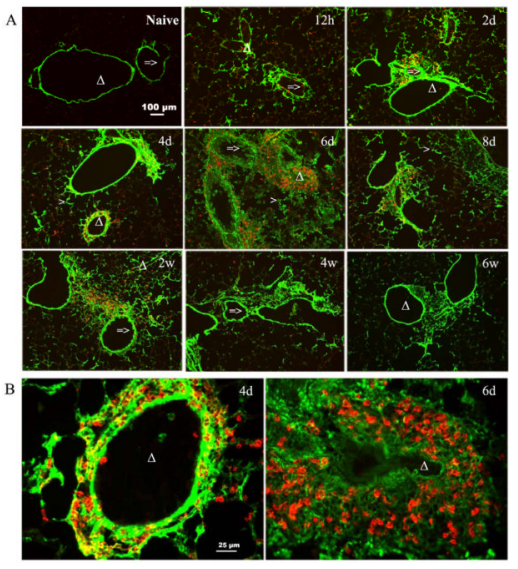

Figure 6. Localization of HA and eosinophils in OCT sections from lungs isolated during acute and chronic stages of antigen exposure

(A) Distributions of HA (stained with HABP, green color) and eosinophils (stained with anti-EMBP, red color) are shown in lung sections representative of the 8 weeks time course of antigen exposure (magnification bar $100 \mu \mathrm{m}$ ). (B) Enlargements (4 times) of lung sections show extensive HA matrix (green) with embedded clusters of eosinophils (red) from 4 days and 6 days (magnification bar $25 \mu \mathrm{m}$ ). Mice were sacrificed at the time points during the course of antigen exposure as in figure 1.

$\Rightarrow$ indicates the airway of lung section.

$\Delta$ indicates the blood vessel of lung section.

$>$ indicates the alveolar interstitium of lung section 

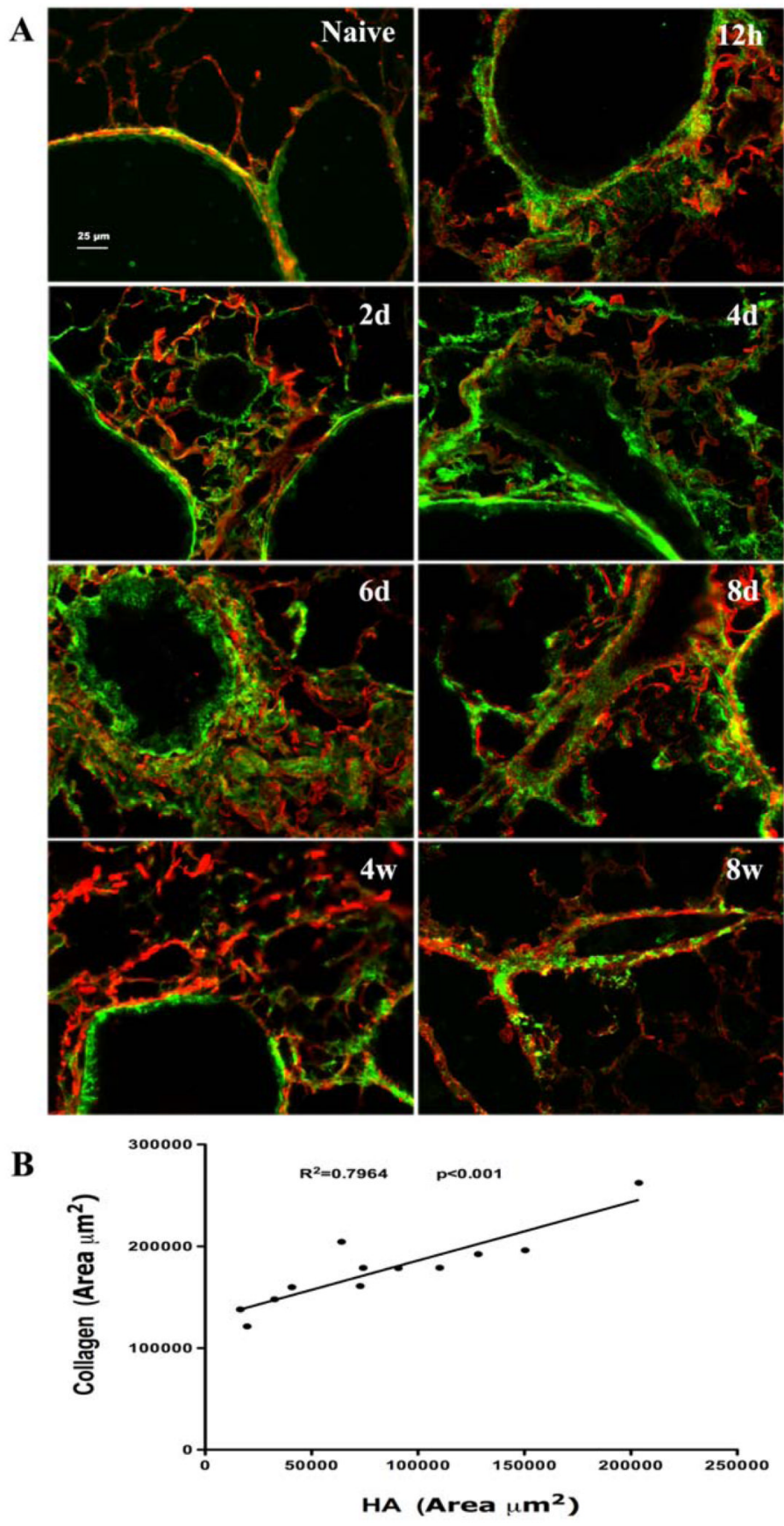

Figure 7. Localization of HA and collagen III in OCT sections from lungs isolated during the acute and chronic stages of antigen exposure

(A) Distributions of HA (stained with HABP, green color) and collagen III (stained with anti-col III, red color) are shown in lung sections representative of the 8 week time course of antigen exposure (magnification bar $25 \mu \mathrm{m}$ ). Additional low magnification fields are shown in supplement figure 4. (B) Correlation between HA and collagen. Mice were sacrificed at the time points during the course of antigen exposure as in figure 1. 
Table 1

Sequences of the primers used for qRT-PCRs

\begin{tabular}{ccl}
\hline Gene \& Symbol & & \multicolumn{2}{l}{ Primer sequences (5'-3') } \\
\hline HA related transcripts & \\
HAS1 & F & GGTGGTTACCGTGTCCAAGTAT \\
& R & GAAGGTAAACTGAGTCCCCAGA \\
HAS2 & F & GGTCCAAGTGCCTTACTGAAAC \\
& R & TGTACAGCCACTCTCGGAAGTA \\
HAS3 & F & AGACCGAGCTAGCCTTCCTAGT \\
& R & TAATGGCCAGATACAGCATGAG \\
HYAL1 & F & GGCCTACCTAGGACTTCCTCAA \\
& R & CTATTCCCGTGACTGTGCCTAT \\
HYAL2 & F & GACCTCAACTACCTGCAGAAGC \\
& R & CCTTATAGTTCCGTTGGCACTG \\
HYAL3 & F & GGAGCATTCACATCCTCTACCT \\
& R & GTCGTCCAGAGACAGGAATCTC \\
\hline
\end{tabular}

